\title{
Generating Functions, Polynomials and Vortices with Alternating Signs in Bose-Einstein Condensates
}

\author{
Anna M. Barry ${ }^{1}$, F. Hajir ${ }^{2}$, P.G. Kevrekidis ${ }^{2}$ \\ ${ }^{1}$ Institute for Mathematics and its Applications, University of Minnesota, \\ Minneapolis, MN 55455 \\ 2 Department of Mathematics and Statistics, University of Massachusetts, \\ Amherst MA 01003-4515, USA
}

\begin{abstract}
In this work, we construct suitable generating functions for vortices of alternating signs in the realm of Bose-Einstein condensates. In addition to the vortex-vortex interaction included in earlier fluid dynamics constructions of such functions, the vortices here precess around the center of the trap. This results in the generating functions of the vortices of positive charge and of negative charge satisfying a modified, so-called, Tkachenko differential equation. From that equation, we reconstruct collinear few-vortex equilibria obtained in earlier work, as well as extend them to larger numbers of vortices. Moreover, particular moment conditions can be derived e.g. about the sum of the squared locations of the vortices for arbitrary vortex numbers. Furthermore, the relevant differential equation can be generalized appropriately in the two-dimensional complex plane and allows the construction e.g. of polygonal vortex ring and multi-ring configurations, as well as ones with rings surrounding a vortex at the center that are again connected to earlier bibliography.
\end{abstract}

\section{Introduction}

The examination of vortex "particles" is a remarkable theme of research in its own right both for smaller and even for larger values of the number of vortices $n$ that has stirred considerable interest in the fluid dynamics engineering, as well as mathematical community. It is in that light that it has been characterized as a "classical mathematics playground" [1. One of the particularly enticing aspects of this research activity has been the connection with the theory of classical (such as the Hermite) and even more modern (so-called Adler-Moser) polynomials. An excellent summary of this link that interweaves applied mathematics with algebra and the theory of polynomials is given in [2]; see also [3, 4].

Recently, a new setting has emerged whereby vortices naturally arise in experiments in quasi-two-dimensional systems. That involves the dynamics of matter waves in ultra-cold atoms and more specifically in the theme of Bose-Einstein condensates (BECs) [5, 6, 7. There, the ability to stir the system (imparting angular momentum), or to quench it (spontaneously locking in vortex patterns), or to drag obstacles through it (producing vorticity through the breakup of superfluidity) enables the spontaneous production of one or more vortices, as has now been summarized in numerous works $8,9,10,11,12$. In fact, experiments have also enabled the production 
of vortices of higher topological charge [13, as well as the generation of robust triangular vortex lattices 14. In the early efforts along this direction, clusters of few vortices were proposed theoretically [15] and created experimentally [16], however the emphasis was chiefly on individual vortices and on large-scale vortex lattices. Recent experimental work, however, has provided a renewed focus on few-vortex clusters [17, 18, 19, 20, 21] through a variety of novel experimental techniques that have offered a more controlled production of such clusters.

It is at the interface of these two fields that the present work treads. In particular, the BEC realm has significant similarities to the earlier fluid vortex setup in that BEC vortices interact in a way that can be accurately approximated by fluid vortex interactions $甘$. However, our setup has also a key additional ingredient affecting the vortex motion, namely the motion of the vortices induced by the (parabolic) magnetic trap. This external potential confining the atoms has been theoretically examined (see 9] for a relevant discussion reviewing the analysis) and experimentally confirmed (see [18] for a recent experimental corroboration) to induce a precession of the vortices around the trap center. The associated precession frequency is nearly constant throughout, roughly, half of the spatial extent of the BEC [21] and for reasons of mathematical simplicity and tractability we will consider it as constant in what follows $\S$.

With the above described setup in mind (combining precession and inter-vortex interactions), we explore the stationary configurations of vortices in BECs both in the case of co-rotating vortices 21] (where only rigidly rotating configurations can be identified as steady in a co-rotating frame; see below) and especially so in that of counter-rotating vortices. The former case reduces to a well-known example from the fluid mechanical case, as analyzed in Section 2 (where it is given as a preliminary calculation along the vein that we follow). However, the counter-rotating case is more complex than its previously analyzed fluid-mechanical sibling due to the presence of the precession term. Hence, there we define two generating functions, one $(P)$ with roots at the positive vortex locations and another $(Q)$ with roots at the negative vortex locations and establish an ordinary differential equation satisfied by a combination of the two for vortices along a real line (section 3; an alternative derivation using a rational distribution function is given in section 4) and within the complex plane (section 6). In section 5, we then use it to produce specific examples of stationary collinear vortex configurations, both ones that are more standard and have been examined before (including in experiments [19, 20]) and ones that have not. In the complex plane case of section 6 , we identify polygonal vortex ring and multi-ring configurations (as well as ones with such rings surrounding a centrally located vortex) and also connect them with earlier numerical observations. Finally, in section 7, we summarize our findings and present a number of relevant conclusions, as well as a series of open problems for future work.

$\ddagger$ This is progressively exact in higher number of atom, more weakly trapped BECs; the inhomogeneous density due to the trapping of BECs weakly screens this interaction, however we will not be concerned with this effect here. An exposition of the screening variation of the vortex interactions can be found in [22] and examples of how this can be captured (by effectively altering the interaction term prefactor) without structurally altering the dynamical terms in the equations of motion can be found e.g. in 20 21].

$\S$ However, as we approach the rims of the BEC, this frequency starts to rapidly increase as shown e.g. in 21]. A relevant discussion in terms of accounting for this increase (and, more generally, a radially dependent frequency) in future work is given in the final section of the present work. 


\section{Setup and a Preliminary Calculation: Collinear Co-rotating Vortices}

In the present study, we briefly touch upon this theme, connecting it to the considerably more complex setting considered herein whereby the vortices feature precession in addition to their pairwise interactions. Perhaps the most elementary yet particularly elegant calculation associated with both settings can be given in the case of $n$ vortices of the same charge, when considering solidly rotating solutions thereof. In that case, the equation of motion reads:

$$
\Omega x_{j}=\sum_{1 \leq i \neq j \leq n} \frac{1}{x_{j}-x_{i}} \quad 1 \leq j \leq n .
$$

In this setting the precession frequency $\Omega$ effectively renormalizes the vortex positions (which scale as $\propto 1 / \sqrt{\Omega}$ ), hence we will set it to unity in what follows. Furthermore, the $\Omega$ term is the one accounting for the rotational frequency of the entire configuration and if an in-trap precession term exists, it can be directly absorbed into this "lumped" precessional term.

The remarkable idea that apparently dates back to the work of Stieljes [23] (subsequently developed by Marden [24, and Szegö [25], among others) was to consider a, so-called, generating function in the form of:

$$
P(x)=\prod_{i=1}^{n}\left(x-x_{i}\right)=\left(x-x_{1}\right) \ldots\left(x-x_{n}\right)
$$

Then, straightforward differentiation leads to the identities:

$$
P^{\prime}(x)=P(x) \sum_{i=1}^{n} \frac{1}{x-x_{i}}, \quad P^{\prime \prime}(x)=P(x) \sum_{i=1}^{n} \sum_{j=1, j \neq i}^{n} \frac{1}{\left(x-x_{i}\right)\left(x-x_{j}\right)}
$$

A key observation then is that the product involved in the second derivative can be simply written as: $\frac{1}{\left(x-x_{i}\right)\left(x-x_{j}\right)}=\left[\frac{1}{x-x_{i}}-\frac{1}{x-x_{j}}\right] \frac{1}{x_{i}-x_{j}}$. But then the sum over $j$ in the term $\frac{1}{\left(x-x_{i}\right)\left(x_{i}-x_{j}\right)}$ can be performed because it is equal to $x_{i} /\left(x-x_{i}\right)$ according to Eq. (11). The second bracket is handled in the same manner (the summation over $i$ is performed) and exchanging indices we get $P^{\prime \prime}(x)=2 P(x) \sum \frac{x_{i}}{x-x_{i}}$. Of course this is true only provided that the points $x_{i}$ are the equilibrium vortex configuration locations. Then, rewriting $x_{i}=-\left(x-x_{i}\right)+x$, we obtain a summation of $(-)$ unity (yielding a factor of $-n$ as multiplying $2 P(x)$ ), as well as a factor of $2 x P^{\prime}(x)$, from the identity of Eq. (3) for $P^{\prime}(x)$. Combining all the above, the differential equation

$$
P^{\prime \prime}(x)=-2 n P(x)+2 x P^{\prime}(x)
$$

arises for the generating function which is exactly the differential equation satisfied by the $\mathrm{n}$-th order Hermite polynomial. In light of the above analysis, this implies that the roots of a rotating equilibrium configuration involving $n$ vortices will lie exactly at the root locations of the $n$-th order Hermite polynomial.

\section{Generating Function ODE for Collinear Counter-rotating BEC Vortices}

We now turn to a variant of this analysis that is relevant to our setting involving vortices also featuring precession. Perhaps the most appropriate context for illustrating relevant ideas is the case of equilibria of precessing vortices involving 
opposite charges [26]. In this case, we will start by assuming that there are $n_{+}$ vortices of one charge and $n_{-}$vortices of the other charge.

Without loss of generality, we record the position of negative charges with an odd index and the position of the positive charges with an even index, so that $x_{j}$ has charge $(-1)^{j}$ for all $j$. Then, the steady state equations for the "odd" charges reads:

$$
x_{2 k+1}=\sum_{l=1}^{n_{+}} \frac{1}{x_{2 k+1}-x_{2 l}}-\sum_{l=1, l \neq k}^{n_{-}} \frac{1}{x_{2 k+1}-x_{2 l+1}} .
$$

Similarly for the "even" positive charges, we will have

$$
x_{2 k}=-\sum_{l=1, l \neq k}^{n_{+}} \frac{1}{x_{2 k}-x_{2 l}}+\sum_{l=1}^{n_{-}} \frac{1}{x_{2 k}-x_{2 l+1}} .
$$

Notice that here these are genuine equilibrium (i.e., stationary) configurations rather than rotating ones, as the opposite actions of precession and interaction can "balance" each other out.

Then, we can define $P(x)=\prod_{l=1}^{n_{+}}\left(x-x_{2 l}\right)$ and $Q(x)=\prod_{l=1}^{n_{-}}\left(x-x_{2 l+1}\right)$, which satisfy similar identities to Eq. (3) [since these do not involve in any way the equations of motion]. Using then the same partial fraction decomposition as above and the equations of motion (5) and (6), we obtain:

$$
\begin{aligned}
& P^{\prime \prime}(x)=2 P(x)\left[\sum_{l=1}^{n_{+}} \frac{-x_{2 l}}{x-x_{2 l}}+\sum_{l=1}^{n_{+}} \sum_{k=1}^{n_{-}} \frac{1}{\left(x-x_{2 l}\right)\left(x_{2 l}-x_{2 k+1}\right)}\right] \\
& Q^{\prime \prime}(x)=2 Q(x)\left[\sum_{k=1}^{n_{-}} \frac{-x_{2 k+1}}{x-x_{2 k+1}}+\sum_{l=1}^{n_{+}} \sum_{k=1}^{n_{-}} \frac{1}{\left(x-x_{2 k+1}\right)\left(x_{2 k+1}-x_{2 l}\right)}\right] .
\end{aligned}
$$

Multiplying $Q \times$ (77) $+P \times(8)$, and once again reshuffling according to our well-known by now partial fraction identity, we retrieve a differential equation combining the generating functions $P$ and $Q$ in the form:

$$
P Q^{\prime \prime}+Q P^{\prime \prime}=2 P^{\prime} Q^{\prime}+2\left(n_{+}+n_{-}\right) P Q-2 x(P Q)^{\prime} .
$$

Remarkably, such equations in the simplest setting of vortices purely interacting (but not precessing) had been developed previously stemming already from the work of Tkachenko in 1964; see the relevant discussion in 22. However, the more standard case of purely interacting vortices provides a much simpler form of Eq. (9) as $P^{\prime \prime} Q+P Q^{\prime \prime}=2 P^{\prime} Q^{\prime}$. An elegant derivation of this latter form also appears in the work of 27 .

We should additionally note here that in the above calculation we labeled the vortices as even and odd implicitly suggesting that the number of the former and of the latter is either equal or differs by one. However, it is straightforward to observe that nowhere in the above derivation is this a crucial assumption and in principle the methodology applies for all vortex numbers, hence so does Eq. (9). Nevertheless, it is important to point out that for vortices of equal charge magnitude (as considered here), we have been unable to find such solutions in our special case examples considered below (e.g. with 3 vortices of one charge and 1 of the other, or 4 of one and 1 of the other etc.). Hence, we will not consider such configurations further here and instead will turn, in section 6 , to special case examples of either equal or differing by one vortex numbers of opposite charges. However, there will also be in that section a notable example of $n$ vortices of unit charge and one vortex of a different opposite (i.e., not equal and opposite) charge. 


\section{An Alternative Rational Function Derivation}

Before turning to examples that illustrate the findings of the previous section, it might be useful for further investigations to record an alternative derivation of the key equation of section 3 which uses a rational function as opposed to a polynomial.

Suppose $s_{1}, \ldots, s_{n}$ are non-zero integers and $x_{1}, \ldots, x_{n}$ are distinct real numbers satisfying for each $j \in\{1,2, \ldots, n\}$,

$$
\sum_{1 \leq i \neq j \leq n} \frac{s_{i}}{x_{i}-x_{j}}=\epsilon s_{j} x_{j}
$$

where we consider $\epsilon= \pm 1$. In this formulation, the $\epsilon=+1$ case is relevant for the realm of co-rotating vortices as per Eq. (11), when all $s_{i}=1$. The $\epsilon=-1$ sign case is the proper one for the case of stationary (non-rotating) configurations of counterrotating vortices, as per Eqs. (5)-(6). More specifically, if we set $s_{i}=(-1)^{i}$ for all

$i$, we are exactly in the situation of (5) and (6) of section 3. Consider the rational function

$$
R(x)=\prod_{i=1}^{n}\left(x-x_{i}\right)^{s_{i}}
$$

By logarithmic differentiation, we have

$$
R^{\prime}(x)=R(x) \sum_{i=1}^{n} \frac{s_{i}}{x-x_{i}} .
$$

Differentiating the above equation and dividing by $R(x)$, we find

$$
\begin{aligned}
\frac{R^{\prime \prime}(x)}{R(x)} & =\frac{R^{\prime}(x)}{R(x)} \sum_{i=1}^{n} \frac{s_{i}}{x-x_{i}}-\sum_{i=1}^{n} \frac{s_{i}}{\left(x-x_{i}\right)^{2}} \\
& =\sum_{1 \leq i \neq j \leq n} \frac{s_{i} s_{j}}{\left(x-x_{i}\right)\left(x-x_{j}\right)}+\sum_{i=1}^{n} \frac{s_{i}^{2}-s_{i}}{\left(x-x_{i}\right)^{2}} .
\end{aligned}
$$

Applying the standard partial fraction decomposition to the first sum on the right hand side above, and using (10), we find after a small calculation that

$$
\begin{aligned}
\frac{R^{\prime \prime}(x)}{R(x)} & =\epsilon 2 \sum_{i=1}^{n} \frac{s_{i}^{2} x_{i}}{x-x_{i}}-\sum_{i=1}^{n} \frac{s_{i}^{2}-s_{i}}{\left(x-x_{i}\right)^{2}} . \\
& =\epsilon\left(-2 n+2 x \sum_{i=1}^{n} \frac{s_{i}^{2}}{x-x_{i}}\right)-\sum_{i=1}^{n} \frac{s_{i}^{2}-s_{i}}{\left(x-x_{i}\right)^{2}} .
\end{aligned}
$$

For the $\epsilon=1$ sign case and $s_{i}=1$ for all $i$, when equation (10) reduces simply to (11), then $R(x)$ is a polynomial with $n$ distinct roots, and we recover $R^{\prime \prime}(x) / R(x)=$ $-2 n+2 x \sum_{i=1}^{n} \frac{1}{x-x_{i}}$, in agreement with our derivation of section 2 .

Hereafter, we focus on the $\epsilon=-1$ sign case, per the configurations of section 3 , involving counter-rotating vortices. Here $R$ is not a polynomial, and we proceed to separate it into its numerator and denominator, writing $R(x)=P(x) / Q(x)$ with polynomials

$$
P(x)=\prod_{1 \leq i \leq n: s_{i}>0}\left(x-x_{i}\right)^{s_{i}}, \quad Q(x)=\prod_{1 \leq i \leq n: s_{i}<0}\left(x-x_{i}\right)^{-s_{i}} .
$$


Note that the convention of the previous section fits into this setup simply with the assignment $s_{i}=(-1)^{i}$ for all $i$. If we let $Y(x)=P(x) Q(x)$, then (11) above can be written in a compact form, namely

$$
\frac{R^{\prime \prime}(x)}{R(x)}=2 n-2 x \frac{Y^{\prime}}{Y}+2\left(\frac{Q^{\prime}}{Q}\right)^{\prime} .
$$

We expand the right hand side and multiply both sides by $P Q$ to find (using also that $\left.Y^{\prime \prime}=P^{\prime \prime} Q+2 P^{\prime} Q^{\prime}+P Q^{\prime \prime}\right)$ finally that

$$
Y^{\prime \prime}+2 x Y^{\prime}-2 n Y=4 P^{\prime} Q^{\prime}
$$

This is the same modified Tkachenko equation as obtained in section 3 .

\section{Special Case Examples}

Let us now seek special case example solutions of Eq. (9) to explore the power of the generating function formalism in comparison to the brute force calculations, based on the equations of motion in [26. The simplest case to examine is that of monomials with $P(x)=(x-a)$ and $Q(x)=(x+a)$, as per the vortex dipole solution examined previously; see also the recent experiments of [18, 20]. In this case, we have $Q(x)=-P(-x)$; in fact, more generally for even vortex number with $n_{+}=n_{-}=n$, we have that $Q(x)=(-1)^{n} P(-x)$, based on the symmetries discussed in [26]. A direct substitution of $P$ and $Q$ into Eq. (9) yields immediately $a= \pm 1 / \sqrt{2}$. This, in turn, implies that $P(x) Q(x)=\left(x^{2}-a^{2}\right)=\left(x^{2}-1 / 2\right) \equiv H_{2}(x)$ i.e., it appears that the Hermite polynomials resurface, albeit now in the form of $P(x) Q(x)$. Unfortunately, this is only a fortuitous coincidence, as in this case of monomials $P^{\prime \prime}=Q^{\prime \prime}=0$ and hence one can proceed to show that

$$
(P Q)^{\prime \prime}=2 P^{\prime} Q^{\prime}=2 x(P Q)^{\prime}+4 P Q .
$$

This, in turn, establishes that $P Q=H_{2}(x)$ according to the equation for the relevant polynomials (cf. Eq. (44)).

In the case of odd $n_{+}+n_{-}$, the above symmetry of the generating functions is broken and we have e.g. for $n_{+}=1$ and $n_{-}=2$ that $P(x)=x$ and $Q(x)=x^{2}-a^{2}$. Once again, a very straighforward direct substitution yields $a= \pm 1 / \sqrt{2}$.

On the other hand, for $n_{+}=n_{-}=2$, we have a choice of $P(x)=(x-a)(x-b)$ and $Q(x)=(x+a)(x+b)$. In this case, direct substitution of the $P$ and $Q$ in Eq. (9) yields the algebraic conditions:

$$
a^{2}+b^{2}=1, \quad a b=\frac{1 \pm \sqrt{2}}{2}
$$

which is in line with the corresponding finding of [26].

In the case of $n_{+}=3$ and $n_{-}=2$, the generating functions assume the following form $P(x)=x\left(x^{2}-a^{2}\right)$ and $Q(x)=x^{2}-b^{2}$. Here, the direct substitution yields anew a system for $a, b$ according to:

$$
a^{2}+b^{2}=1, \quad 2 a^{2}-6 b^{2}+8 a^{2} b^{2}=0 .
$$

This, in turn, results in $a^{2}=\sqrt{3} / 3$ and $b^{2}=(2-\sqrt{3}) / 2$, which we again find to be in agreement with [26. 
The last two cases that we wish to consider following this method are $n_{+}=3$ and $n_{-}=3$, as well as $n_{+}=3$ and $n_{-}=4$. In the former case, $P(x)=(x-a)(x-b)(x-c)$ and $Q(x)=-P(-x)$. This results in three algebraic equations of the following form:

$$
\begin{array}{ll}
a^{2}+b^{2}+c^{2} & =\frac{3}{2} \\
12 a^{2} b^{2} c^{2}-2\left(a^{2} b^{2}+a^{2} c^{2}+b^{2} c^{2}\right)-8 a b c(a+b+c) & =0 \\
4(a+b+c)^{2}-8\left(a^{2} b^{2}+b^{2} c^{2}+a^{2} c^{2}\right) & =0
\end{array}
$$

The solution of the algebraic system of Eqs. (15)-(17) is $a=0.1623, b=-0.4765$ and $c=1.1165$, which indeed when scaled using $\Omega=1 / 2$ as in [26], yields values identical to the ones reported therein.

Finally, the case of $n_{+}=3$ and $n_{-}=4$ yields $P(x)=x\left(x^{2}-a^{2}\right)$ and $Q(x)=\left(x^{2}-b^{2}\right)\left(x^{2}-c^{2}\right)$. Here, the algebraic equations yield:

$$
\begin{array}{ll}
a^{2}+b^{2}+c^{2} & =\frac{3}{2} \\
4\left(b^{2}+c^{2}-a^{2}\right)-8\left(a^{2} b^{2}+a^{2} c^{2}+b^{2} c^{2}\right) & =0 \\
12 a^{2} b^{2} c^{2}-2\left(a^{2} b^{2}+a^{2} c^{2}-3 b^{2} c^{2}\right) & =0 .
\end{array}
$$

These yield $a=0.5546, b=0.2594$ and 1.0607 (again potentially scalable by $\sqrt{1 / \Omega}$ ).

For reasons of completeness, it is relevant to add here a discussion about the stability and observability of such configurations. Among the above aligned vortex configurations, the only one that is dynamically robust and observed experimentally as such is the vortex dipole with $n_{+}=n_{-}=1$; see e.g. [18, 20] and references therein. Dipole dynamics (although not at steady state) have been also experimentally observed in [17. All higher aligned configurations, even though stationary, are, in fact, dynamically unstable, as has been illustrated through detailed numerical bifurcation computations in [28. This is a direct byproduct of their emergence through a cascade of super-critical pitchfork bifurcations from the destabilization of the two-dimensional (planar) analog of a dark soliton. The first such instability (that endows the dark soliton state with one unstable mode) produces as a "daughter branch" the stable vortex dipole. At the next instability, the vortex tripole emerges $\left(n_{+}=1\right.$ and $n_{-}=2$ or equivalently $n_{+}=2$ and $n_{-}=1$ ), although the already present instability of the dark soliton endows it already with an unstable eigenmode, while the dark soliton itself now possesses two unstable eigenmodes past the bifurcation point. Then the cascade continues by producing the aligned quadrupole with $n_{+}=2$ and $n_{-}=2$ which will now inherit the dark soliton's two unstable modes, while the dark soliton gets endowed with a third one and so on and so forth. However, it should be pointed out that despite the instability of all higher states with $\max \left(n_{+}, n_{-}\right)>1$, these states are still potentially observable as long-lived metastable states. An example of this type is the vortex tripole which has been observed as a long-lived state in the experiments of [19]. Additionally, techniques such as the ones of [17, of laser beams creating potential barriers can be utilized in order to create (and trap) different numbers of vortices within the condensates at will [29. Along that experimental vein, one can straightforwardly envision producing stationary configurations of higher $n$ and observing their dynamics (and unstable evolution). As an additional possibility of potential experimental interest, it should be mentioned that such aligned vortex configurations have also been identified in anisotropic settings [30. There, depending on the strength of the anisotropy in the transverse (to the line bearing the vortices) 
direction, it has been demonstrated to be possible to stabilize configurations with arbitrarily many aligned counter-rotating vortices.

It is interesting to add here that observation of the above patterns of algebraic equations suggests that it may be possible to derive general algebraic conditions that the roots of the polynomials may satisfy irrespectively of $n_{+}$and $n_{-}$. For instance, we can see that both for $n_{+}=2=n_{-}$and for $n_{+}=3=n_{-}+1$, it is true that $\sum_{i=1}^{n_{+}+n_{-}} x_{i}^{2}=1$, while the same sum becomes $3 / 2$ for $n_{+}=3=n_{-}$and for $n_{+}=4=n_{-}+1$. Motivated by this finding, we have more generally examined the resulting powers of the polynomials arising in both the left and the right hand side of Eq. (9). As a result, we have found that at power $x^{n_{+}+n_{-}-2}$, the prefactors of the two sides are respectively $n_{+}\left(n_{+}-1\right)+n_{-}\left(n_{-}-1\right)$ and $-4 \sum_{i=1}^{n_{+}+n_{-}} x_{i}^{2}+2 n_{+} n_{-}$, leading eventually to the identity:

$$
\sum_{i=1}^{n_{+}+n_{-}} x_{i}^{2}=\frac{1}{4}\left[2 n_{+} n_{-}-n_{+}\left(n_{+}-1\right)-n_{-}\left(n_{-}-1\right)\right] .
$$

Such identities (verified by all of our above considered cases) have also been considered in the absence of precession in 2 (see e.g. Eq. (10) therein), although they are obtained at the level of the dynamical equations for the vortices rather than those of the generating function ODEs.

\section{Generalizing the Tkachenko Equation in the Complex Plane}

Among the numerous aspects that are worthy of additional investigation in the realm of oppositely charged vortices in the presence of precession, arguably, one of the most important concerns the generalization of the above considerations to states that are not collinear. Hence, we now turn our attention to generalizing the above considerations of our modified Tkachenko equation to the two-dimensional complex plane.

In this case, if we take $n_{+}$vortices $z_{1}, . ., z_{n_{+}}$with charge +1 and $n_{-}$vortices $\zeta_{1}, \ldots, \zeta_{n_{-}}$with charge -1 the relevant stationary equations will be of the form:

$$
\begin{aligned}
& z_{j}=-\sum_{k=1, k \neq j}^{n_{+}} \frac{z_{j}-z_{k}}{\left|z_{j}-z_{k}\right|^{2}}+\sum_{k=1}^{n_{-}} \frac{z_{j}-\zeta_{k}}{\left|z_{j}-\zeta_{k}\right|^{2}} \\
& \zeta_{j}=-\sum_{k=1, k \neq j}^{n_{-}} \frac{\zeta_{j}-\zeta_{k}}{\left|\zeta_{j}-\zeta_{k}\right|^{2}}+\sum_{k=1}^{n_{+}} \frac{\zeta_{j}-z_{k}}{\left|\zeta_{j}-z_{k}\right|^{2}} .
\end{aligned}
$$

We again define the $P$ and $Q$ generating functions in the same way as before as $P(z)=\prod_{i=1}^{n_{+}}\left(z-z_{i}\right)$ and $Q(z)=\prod_{j=1}^{n_{-}}\left(z-\zeta_{j}\right)$. While Eqs. (3) remain the same in this case, the main change arises at the level of Eqs. (7)-(8). Here, the identities become

$$
\begin{aligned}
& P^{\prime \prime}(z)=P(z) \sum_{i=1}^{n_{+}} \frac{2}{z-z_{i}}\left[-\bar{z}_{i}+\sum_{k=1}^{n_{-}} \frac{1}{z_{i}-\zeta_{k}}\right] \\
& Q^{\prime \prime}(z)=Q(z) \sum_{j=1}^{n_{-}} \frac{2}{z-\zeta_{j}}\left[-\bar{\zeta}_{j}+\sum_{k=1}^{n_{+}} \frac{1}{\zeta_{j}-z_{k}}\right]
\end{aligned}
$$

In a way paralleling the derivation of Eq. (9), we form $P^{\prime \prime}(z) Q(z)+Q^{\prime \prime}(z) P(z)$. In that context, the second term in the brackets within Eqs. (24)-(25) will again yield $2 P^{\prime}(z) Q^{\prime}(z)$, but the first term is more complicated. In particular, $\bar{z}_{j}=x_{j}-i y_{j}=z_{j}-$ 
$2 i y_{j}$ and $\bar{\zeta}_{j}=\zeta_{j}-2 i v_{j}$. The first term (involving $z_{j}$ and $\zeta_{j}$ ) is now entirely analogous to the real case and will provide the terms $2\left(n_{+}+n_{-}\right) P(z) Q(z)-2 z(P(z) Q(z))^{\prime}$. However, the last term provides an additional term $4 i P(z) Q(z)\left(\sum_{j=1}^{n_{+}} \frac{y_{j}}{z-z_{j}}+\sum_{j=1}^{n_{-}} \frac{v_{j}}{z-\zeta_{j}}\right)$ (cf. the term of the right hand side of Eq. (12) of [2]). The resulting final form of the modified Tkachenko equation is:

$$
\begin{aligned}
P^{\prime \prime} Q+P Q^{\prime \prime} & =2 P^{\prime} Q^{\prime}+2\left(n_{+}+n_{-}\right) P Q-2 z(P Q)^{\prime} \\
& +4 i P Q\left(\sum_{j=1}^{n_{+}} \frac{y_{j}}{z-z_{j}}+\sum_{j=1}^{n_{-}} \frac{v_{j}}{z-\zeta_{j}}\right)
\end{aligned}
$$

Generally, Eq. (26) is far more difficult to solve than Eq. (9). However, relevant results can still be extracted from it in some special case examples. Perhaps a prototypical one is that of two interlaced polygonal vortex rings (with the same radius) consisting of oppositely charged vortices. Non-stationary variants of such configurations were observed to spontaneously emerge through the instability of ring dark solitons in 31. Stationary variants of such states were identified in 32 and a more systematic bifurcation analysis thereof connecting them to the instabilities of the ring dark soliton was given in [33. An analysis of the existence and stability of these configurations for arbitrary total number of vortices $n_{+}+n_{-}$was given in [26]. It was found there that the only (non-collinear) such configuration which is stable at the "particle level" is the so-called vortex quadrupole [34; see also [28. On the other hand, configurations such as the vortex hexagon, octagon, decagon, etc. are always unstable, arising through a cascade of progressive bifurcations from the ring dark soliton. Interestingly, this is an entirely analogous process to the cascade of instabilities imparted on the collinear vortex states by the rectilinear dark soliton. This imparts additional unstable modes to the waveform as the number of vortex increases i.e., the hexagon has always at least one unstable mode, the octagon at least two, the decagon at least three and so on. Moreover, the polygonal ring states are absent for the case where $n_{+}+n_{-}$is odd [26]. Now, we turn to the identification of such states through the Tkachenko equation approach of Eq. (26).

We assume that $n_{+}=n_{-} \equiv n$ and then the two polygonal rings, possessing the same radius can be represented in the complex plane by $P(z)=z^{n}-R^{n}$ and $Q(z)=z^{n}-R^{n} e^{i n \phi}$. In the second expression the roots (i.e., vortex locations) are assumed to be (uniformly) shifted by an angle $\phi$. This achieves the interlaced vortex ring configuration of interest. Direct substitution of the $P$ and $Q$ in Eq. (26) yields numerous cancelations and only two conditions, due to the vanishing of the polynomial prefactors of $z^{2 n-2}$ and of $z^{n-2}$ [it should be added as an aside that properties of the complex roots of unity and geometric series are used in order to evaluate the last term in Eq. (26); cf. Eq. (13) of [2]]. These two conditions are:

$$
\begin{aligned}
& R^{2}=\frac{1}{2} \\
& e^{i n \phi}=-1
\end{aligned}
$$

The former condition represents an algebraic constraint either on the radius of the vortex ring or, equivalently (for arbitrary vortex ring radius) on the precession frequency (cf. with [26]). The latter condition, however, is arguably even more important as it suggests that the two rings are displaced with respect to each other by an angle of $\phi=\pi / n$. This is consonant with the numerical observations (also of the corresponding PDE model), as e.g. in the case of the vortex quadrupole [34, 28, 
of $n=2$, the rotation is by $\pi / 2$, in that of the hexagon [32, 33] of $n=3$, it is by $\pi / 3$ and so on. Moreover, since the vortices of a single charge are rotated with respect to their neighbors of the same sign by $2 \pi / n$ (due to the structure of the complex roots of unity), this suggests that in the interlaced polygonal configuration, (as expected by symmetry) the vortices are positioned in an alternating charge fashion at equal angles of $\pi / n$.

Beyond the single vortex polygon one can consider two or more nested polygons. Two rings, each possessing 4 vortices, were observed numerically in the PDE model as bifurcating from the $\mathrm{X}$-shaped dark soliton cross 33. Like in the case of a single polygon, we find that there are no fixed equilibria consisting of nested polygons with odd numbers of vortices. With this as motivation we investigate two polygons (each interlaced as above) having an equal, even number of vortices. We consider Eq. (26) with

$$
\begin{aligned}
& P(z)=\left(z^{n}-R_{1}^{n}\right)\left(z^{n}-R_{2}^{n} e^{i n \phi}\right) \\
& Q(z)=\left(z^{n}-R_{2}^{n}\right)\left(z^{n}-R_{1}^{n} e^{i n \phi}\right)
\end{aligned}
$$

and obtain a polynomial in $z$ which, after factoring out $z^{n-2}$, has only four (potentially) nonzero terms:

$$
\begin{aligned}
& 4 n\left(-1+R_{1}^{2}+R_{2}^{2}\right) z^{4 n} \\
& -n\left(1+e^{i n \phi}\right)\left(R_{2}^{n}\left(-3+n+4 R_{1}^{2}+2 R_{2}^{2}\right)+R_{1}^{n}\left(-3+n+2 R_{1}^{2}+4 R_{2}^{2}\right)\right) z^{3 n} \\
& +2 n e^{i n \phi}\left(\left(-1+2 R_{1}^{2}\right) R_{2}^{2 n}+R_{1}^{2 n}\left(-1+2 R_{2}^{2}\right)\right. \\
& +2 R_{1}^{n} R_{2}^{n}\left(-1+2 n+R_{1}^{2}+R_{2}^{2}+\left(-1+R_{1}^{2}+R_{2}^{2}\right) \cos (n \phi)\right) z^{2 n} \\
& -e^{i n \phi}\left(1+e^{i n \phi}\right) n R_{1}^{n} R_{2}^{n}\left(\left(-1+n+2 R_{1}^{2}\right) R_{2}^{n}+R_{1}^{n}\left(-1+n+2 R_{2}^{2}\right)\right) z^{n} .
\end{aligned}
$$

Setting the first expression equal to zero we find the relationship between the two radii $R_{1}^{2}+R_{2}^{2}=1$. Using this relationship and noting that $n \geq 2$ forces us to choose $\phi=\pi / n$ from the second term. This choice also renders the fourth term identically zero, and we now have two equations which can be used to determine admissible radii for the rings, depending on the particular value of $n$ :

$$
\begin{aligned}
& 1=R_{1}^{2}+R_{2}^{2} \\
& 0=4 n R_{1}^{n} R_{2}^{n}+\left(-1+2 R_{1}^{2}\right) R_{2}^{2 n}+R_{1}^{2 n}\left(-1+2 R_{2}^{2}\right) .
\end{aligned}
$$

Interestingly, we are able to modify the forms of $P$ and $Q$ slightly to deduce the existence of another family of equilibria made up of two interlaced $N$-gons with different radii and a vortex at the center of the configuration. A configuration from this family can be found in 33. Figure 6(b), upper left. Without loss of generality, we choose the vortex at the center to have charge +1 and set

$$
\begin{aligned}
& P(z)=z\left(z^{n}-R_{1}^{n}\right) \\
& Q(z)=\left(z^{n}-R_{2}^{n} e^{i n \phi}\right) .
\end{aligned}
$$

Now, Eq. (26) is a polynomial with two nonzero coefficients corresponding to $z^{n}$ and $z^{n-1}$. The coefficients are zero provided

$$
\begin{aligned}
& 1=R_{1}^{2}+R_{2}^{2} \\
& 0=-e^{i n \phi}\left(1+n+2 R_{1}^{2}\right) R_{2}^{n}-R_{1}^{n}\left(-3+n+2 R_{2}^{2}\right) .
\end{aligned}
$$

By dividing the second equation into real and imaginary parts, we again find $\phi=\frac{\pi}{n}$. For a given $n$ the remaining equations can again be used to determine $R_{1}$ and $R_{2}$. Both the previous and this configuration are generalizations and analytical 
characterizations of specific example solutions identified at the level of the partial differential equation (the so-called Gross-Pitaevskii model) describing the mean-field atomic BEC system [33] for particular $n$ 's $(n=2$ for the former, and $n=4$ for the latter case).

There exists one more case in which we were also able to generalize the calculation in the complex plane. This pertains to the situation where a single vortex (to be eventually placed at the origin) is surrounded by vortices of (what we will see has to be by necessity, if the configuration is to be stationary) opposite charge. It is noteworthy that this case can also be captured by Eq. (26) upon suitable modification (to encompass the general charge at the origin and the choice of $Q(z)=z$ ), however, we will develop the relevant case directly from the stationary equation for the system of a single vortex of charge $S_{0}$ located at $z_{0}$, surrounded by $n$ vortices of charge $S$ located on a ring of radius $R$. In that case, assuming a unit frequency of precession, we have that:

$$
\begin{aligned}
& S_{0} z_{0}=-\sum_{j=1}^{n} \frac{S}{\bar{z}_{0}-\bar{z}_{j}} \\
& S z_{k}=-\sum_{j=1, j \neq k} \frac{S}{\bar{z}_{k}-\bar{z}_{j}}-\frac{S_{0}}{\bar{z}_{k}-\bar{z}_{0}}
\end{aligned}
$$

We define as previously $P(z)=\prod_{j=1}^{n}\left(z-z_{j}\right)$, having as before that $P^{\prime \prime}(z)=$ $2 P(z) \sum_{i=1}^{n} \sum_{j=1, j \neq i} \frac{1}{z-z_{i}} \frac{1}{z_{i}-z_{j}}$. Performing the step that is by now fairly familiar, the summation over $j$ can be substituted (from Eq. (39)) yielding:

$$
P^{\prime \prime}(z)=2 P(z) \sum_{i=1}^{n} \sum_{j=1, j \neq i} \frac{1}{z-z_{i}}\left[-\frac{S_{0}}{S} \frac{1}{z_{i}-z_{0}}-\bar{z}_{i}\right]
$$

The last term will yield familiar contributions in the equation leading to terms (cf. Eq. (26) ): $2 n P(z)-2 z P^{\prime}(z)+4 i P(z) \sum_{n} y_{i} /\left(z-z_{i}\right)$. On the other hand, the contribution of the first term is less familiar and amounts to $-2 P(z)\left(S_{0} / S\right) \sum_{i=1}^{n}(z-$ $\left.z_{i}\right)^{-1}\left(z_{i}-z_{0}\right)^{-1}$. Using the fact that $P(z)=z^{n}-R^{n}$ amounting to a ring of $n$ vortices of charge $S$ surrounding the central one of charge $S_{0}$, all terms other than then one proportional to $S_{0} / S$ in Eq. (40) can be computed. The term $\propto S_{0} / S$ can be computed, however, as well upon the additional assumption that $z_{0}=0$, which is also meaningful from the point of view of the configuration's symmetry. In that case, we have that:

$$
-2 P(z) \frac{S_{0}}{S} \sum_{i=1}^{n} \frac{1}{z-z_{i}} \frac{1}{z_{i}-z_{0}}=-2 P(z) \frac{S_{0}}{S} \frac{n z^{n-2}}{z^{n}-R^{n}}=-2 n \frac{S_{0}}{S} n z^{n-2}
$$

Using this identity and the form of $P(z)$, direct substitution in Eq. (40) yields [in addition to numerous cancellations] the formula:

$$
R^{2}=-\frac{S_{0}}{S}+\frac{1-n}{2}
$$

From this, we naturally infer that $S_{0}$ and $S$ must be oppositely charged i.e. $S_{0} S<0$ for such a stationary configuration to exist. This is also intuitively clear, as otherwise (if all charges were of the same sign), the configuration would tend to rigidly rotate rather than be at equilibrium. In fact, since the contribution of $(1-n) / 2$ is negative, what Eq. (42) suggests is that not only should $S_{0}$ be of opposite sign than $S$, but also of sufficiently high charge, so as to effectively counter the rotational tendency of 
the charges $S$. I.e., the relevant equilibrium condition, in addition to the charge sign inequality above, yields $\left|S_{0} / S\right|>(n-1) / 2$. It is interesting to note here that a special case of this configuration with $n=4$ was encountered previously in [26] (see Eq. (13) therein), as well as numerically analyzed (again for $n=4$ ) in 33, where its instability was corroborated.

While in the above analysis, for reasons of analytical tractability and computational convenience, we have restricted our considerations to the case of symmetric polygonal configurations, there exist numerous intriguing examples of additional stationary configurations. More specifically, as can be seen in 35] for the case where the precession term is absent, there are numerous configurations that are genuinely asymmetric (cf. [35], Fig. 4 in [2] etc.). It would be of particular interest (although it would most likely be a numerical task) to examine the possibility of such configurations in the present context.

\section{Conclusions and Future Challenges}

In the present work, we extended the considerations of [2] to the realm of BoseEinstein condensates and the cases of vortices precessing therein, assuming a constant precession frequency. As discussed earlier in [36] (see also 37]), the co-rotating case of the BEC problem is tantamount to the well-known fluid case. As a result (after writing the stationary problem in the frame rotating with the vortices), by defining a suitable generating function with roots thereof representing the positions of the vortices, it is found that this function satisfies a Hermite differential equation, indicating that the vortices in such a rigidly rotating configuration are sitting at the location of the well-known roots of Hermite polynomials.

On the other hand, the focus herein has been on the less straightforward case of genuinely stationary configurations consisting of oppositely charged vortices. For this case, we derived the appropriate generalized differential equation, illustrating that while in the case of the dipole, the vortex equilibrium positions are still Hermite polynomial roots, this is not true more generally. We used the relevant differential equation in a host of cases of different (small) numbers of positive and negative charge vortices, to identify the corresponding equilibria consonantly (in all cases that were examined before) to what was known about such roots. We also used the relevant polynomial expressions to derive "moment" conditions about the positions of the vortices in the general case of arbitrary vortex numbers.

While the above considerations were initially set on the line (i.e., collinear equilibria), subsequently, we generalized the configurations to the complex plane and identified relevant additions that needed to be made to the differential equation. Once again profound similarities, but also important differences were illustrated with respect to the classic work of 2] (and references therein). Armed with the relevant differential equation, and despite its complexity, we were able to identify one of the prototypical configurations of vortices in the complex plane well known in the BEC problem. In

particular, we obtained two interlaced vortex polygons, with an equal number of vortices $n$, shifted relative to each other by an angle of $\pi / n$. In a similar fashion we were able to identify two nested, interlaced vortex polygons in cases with and without a vortex at the center of the configuration as well as the so-called $N+1$ vortex solution, where a single vortex of (sufficiently high - relevant conditions were derived-) charge $S_{0}$ is surrounded by vortices of opposite charge $S$. 
There are numerous directions that open up as a result of the analysis and calculations presented herein.

Starting from the end and the considerations in the two-dimensional plane, it would certainly be interesting and relevant to extend these calculations to other configurations. A prototypical case of especially high interest, as highlighted at the end of the previous section, concerns asymmetric stationary configurations. For these, it would be especially interesting, either using the modified Tkachenko equation developed herein and/or numerical approaches to be able to identify such configurations (and, of course, to also get a sense of their potential stability/instabilities).

Furthermore, the exposition of [2] provides yet another particularly interesting avenue of potential analysis concerning the collinear vortices. In particular, it is found that a recursion formula can be set up that provides a direct connection between the polynomials $P_{n-1}, P_{n}$ and $P_{n+1}$ arising for different vortex numbers. This recursion relation is associated (in the absence of precession) to the so-called Adler-Moser polynomials 38 that surprisingly also arise in the study of rational solutions of the Korteweg-de Vries equation. Identifying the relevant recursion relation and connecting it (potentially) to particular polynomial properties would be a separate topic worth investigating in its own right. However, our preliminary calculations suggest that this may not be straightforward. In such a calculation one assumes that the two generating functions $P$ and $Q$ satisfy Eq. (9) and also that $P$ and $R$ are an additional pair satisfying a similar modified Tkachenko equation:

$$
P R^{\prime \prime}+R P^{\prime \prime}=2 P^{\prime} R^{\prime}+2\left(n_{+}+n_{-}\right) P R-2 x(P R)^{\prime},
$$

provided that $R^{\prime} Q-R Q^{\prime}=F(P, x)$. If an $F$ is found such that Eqs. (9) and (43) are concurrently satisfied, then $Q, P$ and $R$ become successive elements in a recursive formula that allows us to build from two of these polynomials, the next one in the sequence of such polynomials (i.e. $Q \sim P_{n-1}, P \sim P_{n}$ and $R \sim P_{n+1}$ and one can then use the recursion to identify $P_{n+2}$ and so on). In the case where Eqs. (91) and (43) only possess the first term of the right hand side (cf. [2]), it is found that $F(P, x)=P^{2}$ and the Adler-Moser polynomials arise as a result of the recursion. Here, a modification of that calculation yields that, for example, $F(P, x)=P^{2} e^{-x^{2}}$ provides such a solution, however the non-polynomial nature of the exponential factor precludes its use for the purposes of a recursive construction of higher order polynomials in this hierarchy. Of course, this result is inconclusive as it does not immediately preclude the presence of such polynomials, but it is perhaps suggestive in that direction. This theme is certainly worthy of further examination, as well.

Finally, all of the above considerations have been developed for a constant precession frequency. Nevertheless, it has been theoretically proposed [9] and experimentally confirmed [18] that as the outer rim of the condensates is approached, the precession frequency in fact increases. Although not accurate for all precession radii, a reasonable approximation of this radial dependence is $\omega=\omega_{0} /\left(1-r^{2}\right)$ [21] (with the radius of precession normalized to the size of the condensate or more concretely to the so-called Thomas-Fermi radius). This dependence suggests the interest and relevance of a potential generalization of the considerations presented herein to a case of a radially dependent precession frequency. It is then natural to expect that even in the collinear vortex case, summation terms (involving the roots) somewhat reminiscent of the ones arising e.g. in Eq. (26) will appear due to the $1 /\left(1-r_{j}^{2}\right)$ term. Nonetheless, the corresponding Tkachenko equation may still be 
useful (at least in the case of small $n$ 's as considered herein) towards the identification of the vortex equilibrium positions.

Studies along these directions are currently in progress and will be reported in future publications.

Acknowledgements: P.G.K. acknowledges support from the National Science Foundation under grants CMMI-1000337, DMS-1312856, from FP7-People under grant IRSES-606096 from the Binational (US-Israel) Science Foundation through grant 2010239, and from the US-AFOSR under grant FA9550-12-10332.

[1] H. Aref, J. Math. Phys. 48, 065401 (2007).

[2] H. Aref, Fluid Dyn. Res. 39, 5 (2007).

[3] H. Aref, P. Newton, M. Stremler, T. Tokieda, D. Vainchtein, Advances in applied Mechanics 39, 1 (2003).

[4] P. Newton, The N-vortex problem: analytical techniques, Springer-Verlag (New York, 2001).

[5] C.J. Pethick and H. Smith, Bose-Einstein condensation in dilute gases, Cambridge University Press (Cambridge, 2002).

[6] L.P. Pitaevskii and S. Stringari, Bose-Einstein Condensation, Oxford University Press (Oxford, 2003).

[7] P.G. Kevrekidis, D.J. Frantzeskakis, R. Carretero-Gonzlez, Emergent Nonlinear Phenomena in BoseEinstein Condensates, Springer-Verlag, Berlin, 2008.

[8] R.J. Donnelly, Quantized Vortices in Helium II, Cambridge University Press, New York, 1991;

[9] A. L. Fetter and A. A. Svidzinsky, J. Phys.: Condens. Matter 13, R135 (2001).

[10] A. L. Fetter, Rev. Mod. Phys. 81, 647 (2009).

[11] P.G. Kevrekidis, R. Carretero-González, D.J. Frantzeskakis and I.G. Kevrekidis, Mod. Phys. Lett. B 18, 1481 (2004).

[12] M. Tsubota, K. Kasamatsu and M. Kobayashi, arXiv:1004.5458

[13] A.E. Leanhardt, A. Görlitz, A.P. Chikkatur, D. Kielpinski, Y. Shin, D.E. Pritchard, W. Ketterle, Phys. Rev. Lett. 89, 190403 (2002); Y. Shin, M. Saba, M. Vengalattore, T.A. Pasquini, C. Sanner, A.E. Leanhardt, M. Prentiss, D.E. Pritchard, W. Ketterle, Phys. Rev. Lett. 93, 160406 (2004).

[14] C. Raman, J.R. Abo-Shaeer, J.M. Vogels, K. Xu, W. Ketterle, Phys. Rev. Lett. 87, 210402 (2001).

[15] Y. Castin, R. Dum, Eur. Phys. J. D 7, 399 (1999).

[16] K.W. Madison, F. Chevy, W. Wohlleben, J. Dalibard, Phys. Rev. Lett. 84806 (2000).

[17] T.W. Neely, E.C. Samson, A.S. Bradley, M.J. Davis, B.P. Anderson, Phys. Rev. Lett. 104, 160401 (2010).

[18] D.V. Freilich, D.M. Bianchi, A.M. Kaufman, T.K. Langin, and D.S. Hall, Science 329, 1182 (2010).

[19] J.A. Seman, E.A.L. Henn, M. Haque, R.F. Shiozaki, E.R.F. Ramos, M. Caracanhas, P. Castilho, C. Castelo Branco, P.E.S. Tavares, F. J. Poveda-Cuevas, G. Roati, K.M.F. Magalhaes, V.S. Bagnato, Phys. Rev. A 82, 033616 (2010).

[20] S. Middelkamp, P. J. Torres, P. G. Kevrekidis, D. J. Frantzeskakis, R. Carretero-González, P. Schmelcher, D. V. Freilich, and D. S. Hall, Phys. Rev. A 84, 011605 (2011).

[21] R. Navarro, R. Carretero-González, P.J. Torres, P.G. Kevrekidis, D.J. Frantzeskakis, M.W. Ray, E. Altuntaş, and D.S. Hall, Phys. Rev. Lett. 110, 225301 (2013).

[22] S. McEndoo, Th. Busch, Phys. Rev. A 79, 053616 (2009).

[23] T.J. Stieljes, Act. Math. 6-7, 321 (1885).

[24] M. Marden, Geometry of polynomials, American Mathematical Society (Providence, 1949).

$[25]$ G. Szegö, Orthogonal polynomials, American Mathematical Society (Providence, 1959).

[26] A.M. Barry, P.G. Kevrekidis, J. Phys. A: Math. Theor. 46, 445001 (2013).

[27] J.B. Kadtke and L.J. Campbell, Phys. Rev. A 36, 4360 (1987).

[28] S. Middelkamp, P. G. Kevrekidis, D.J. Frantzeskakis, R. Carretero-González, and P. Schmelcher Phys. Rev. A 82, 013646 (2010).

[29] This is a technique that has been experimentally developed and tested in the group of Prof. B. Anderson (B.P. Anderson, personal communication).

[30] J. Stockhofe, S. Middelkamp, P.G. Kevrekidis and P. Schmelcher, EPL 93, 20008 (2011). 
[31] G. Theocharis, D. J. Frantzeskakis, P. G. Kevrekidis, B. A. Malomed, and Yu. S. Kivshar, Phys. Rev. Lett. 90, 120403 (2003); see also G. Herring, L. D. Carr, R. Carretero-González, P. G. Kevrekidis, and D. J. Frantzeskakis Phys. Rev. A 77, 023625 (2008).

[32] T. Kapitula, P. Kevrekidis, and R. Carretero-González Phys. D 233, 112 (2007).

[33] S. Middelkamp, P.G. Kevrekidis, D.J. Frantzeskakis, R. Carretero-Gonzlez, and P. Schmelcher Phys. D 240, 1449 (2011).

[34] see e.g. M. Möttönen, S.M.M. Virtanen, T. Isoshima, and M.M. Salomaa, Phys. Rev. A 71, 033626 (2005) and V. Pietilä, M. Möttönen, T. Isoshima, J.A.M. Huhtamäki, and S.M.M. Virtanen, Phys. Rev. A 74, 023603 (2006).

[35] H. Aref and D. Vainchtein, Nature 392, 769 (1998).

[36] Y. Chen, T. Kolokolnikov and D. Zhirov, Proc. Roy. Soc. A 469, 20130085 (2013).

[37] T. Kolokolnikov, P.G. Kevrekidis, R. Carretero-González, Proc R Soc A 470, 20140048 (2014).

[38] M. Adler and J. Moser, Comm. Math. Phys. 61, 1 (1978). 